\section{Neuer monoklonaler Antikörper punktet bei Plaque-Psoriasis}

— Eine große Phase-III-Studie belegt die Wirksamkeit und Verträglichkeit des neuen monoklonalen Antikörpers Ustekinumab (Stelara ${ }^{\circledR}$ ) bei der Behandlung der mittelschweren bis schweren Plaque-Psoriasis. An der Studie, die kürzlich im New England Journal of Medicine veröffentlicht wurde [Griffiths, et al. N Engl J Med 2010; 362: 118-128] haben über 900 Psoriasis-Patienten teilgenommen, die randomisiert entweder 45 oder 90 mg Ustekinumab in den Wochen o und 4 erhielten oder zweimal wöchentlich 50 mg Etanercept über 12 Wochen.

Ustekinumab, ein rein humaner monoklonaler Antikörper, dessen Wirkprinzip auf der Blockade der p40-Untereinheit der Interleukine 12 und 23 beruht, führte innerhalb des 12-wöchigen Beobachtungszeitraums bei 68 bzw. 74\% der Patienten, die 45 oder 90 mg erhalten hatten, zu einem Rückgang der Plaque-Psoriasis um mindestens $75 \%$, gemessen anhand des Psoirasis Area and Severity Index (PASI). Dies war unter den mit dem TNF $\alpha$-Blocker Etanercept Behandelten lediglich bei $57 \%$ der Fall. Generell stellte sich der klinische Therapieerfolg un- ter Ustekinumab schneller ein: Bereits bis zur Woche 8 erzielten hier deutlich mehr Patienten einen PASI 75.

Darüber hinaus zeigten sich auch deutliche Unterschiede im Ausmaß der klinischen Verbesserung: Während 36 respektive $45 \%$ der mit 45 oder 90 mg Ustekinumab Behandelten innerhalb von 12 Wochen ein PASI-90-Ansprechen erreichen (das einer fast vollständigen Abheilung der von der Plaque-Psoriasis betroffenen Hautstellen entspricht), gelang dies nur $23 \%$ der Patienten unter Etanercept.

Patienten, die auf Etanercept nur unzureichend angesprochen hatten, erhielten in den Wochen 16 und 20 jeweils eine subkutane Injektion mit 90 mg Ustekinab. Daraufhin stellten die Prüfärzte bei $49 \%$ dieser Patienten in der Woche 28 eine deutliche Besserung des Hautbefundes (PASI 75) fest.

Die unerwünschten Wirkungen von Etanercept und Ustekinab waren dabei generell vergleichbar.

dka

Nach Informationen von Janssen-Cilag

\section{Forschungspreis} gefällig?

— Nach dem Spiel ist vor dem Spiel: Kaum wurden am 5. Februar dieses Jahres der mit 20.000 Euro dotierte Forschungspreis sowie zwei mit je 4.00o Euro honorierte Publikationspreise der „Fondation Internationale La Roche-Posay 2009“ in Frankfurt verliehen, hat inzwischen die Bewerbungsfrist für die Preisträger 2010 begonnen. Die Preise winken jungen Dermatologen, die in biologischer, pharmakologischer oder klinischer Dermatologie forschen. Die Abgabefrist für die Bewerbung endet am 14. Juni 2010. Über die eingereichten Arbeiten befinden die Juroren im Oktober 2010 im Rahmen der EADVJahrestagung in Göteborg. Weitere Infos unter www.larocheposay.de unter „Dermatologische Expertise“ und „Forschungspreise“.

dka

Nach Informationen von La RochePosay

\title{
Sonnenschutz für Problemhaut
}

- Menschen mit Hautproblemen oder Hauterkrankungen benötigen häufig spezielle Produkte, um ihre Haut adäquat vor übermäßiger Sonnenbestrahlung zu schützen. Das gilt beispielsweise für Patienten mit Akne oder Neurodermitis genauso wie für Personen, die gerade ein Hautpeeling hinter sich haben. Auch ältere Menschen sollten Sonnenschutzpräparate mit ausreichend hohem Lichtschutzfaktor verwenden: „Sie werden alt genug, um ihren Hautschaden noch zu erleben", sagte Dr. Simone Presto von der Firma Beiersdorf in Hamburg.

Für Personen mit Problemhaut gibt es aus der Eucerin ${ }^{\circledR}$-Serie eine Auswahl verschiedener Produkte. Alle Produkte enthalten einen hochwirksamen UVA- und UVB-Filter und werden mit dem Lichtschutzfaktor 50 angeboten. Für trockene Haut steht Eucerin Sun Lotion zur Verfügung, das zusätzlich Gluco-Glycerol zur Aktivierung des hauteigenen Feuchtigkeitshaushaltes enthält. Klinisch untersucht wurde das Produkt u.a. bei Patienten mit Neurodermitis und bei Diabetikern, die häufig eine sehr trockene Haut haben.
Eucerin Sun protection enthält unter anderem das stark wirksame Antioxidans Licochalon A aus der chinesischen Süßholzwurzel. Eucerin Sun Creme ist eine leicht getönte Creme zur Gesichtspflege, das als Sonnenschutz Titandioxid enthält. Das Titandioxid liegt in sehr feinen NanoPartikeln vor.

„Durch die aktuelle Nano-Technologie-Diskussion werden die Verbraucher undifferenziert verunsichert", berichtete Presto. Dabei sei das Nano-Titandioxid im Sonnenschutz gut untersucht: Es erfolgt keine Resorption über die Haut und selbst in hohen Dosen im Tierversuch wurde keinerlei toxische Wirkung gesehen. Mit Sonnenschutzpräparate mit Titandioxid oder Zinkdioxid können sogar Babys eingecremt werden, wie die Expertin betonte.

bk

Sonnen-Symposium Nivea und Eucerin, Hamburg, 812.2009 (Veranstalter: Beiersdorf) 